\title{
The Semantics in GPS Based Mobile Applications for Blind Users Navigation Outdoor
}

\author{
Israh Akbar, Ahmad Fatzilah Misman \\ Dept Information Systems, International Islamic University Malaysia, Kuala Lumpur, Malaysia \\ israakbar1990@gmail.com \\ Dept Information Systems, International Islamic University Malaysia, Kuala Lumpur, Malaysia \\ zidny@ittelkom-pwt.ac.id
}

\begin{abstract}
This research is a study on improving information facilitated by common GPS based mobile application for navigation especially by the blind users. For a blind person, the main source of information is words and how to improve the effectiveness of the explanation using mobile application for them is tested in this research. The long term goal of technology is to create a movable, selfcontained system that allows visually impaired people to navigate through unfamiliar environments without the assistance of guides The experiment for the research took place at MFB (Malaysian Foundation for the Blind) using existing GPS based mobile applications and the results were used to prove the hypothesis that the blind are at a considerable downside because of the absence of information and providing a detailed description of the environment could be used in the GPS navigation applications for the blind pedestrians to help them navigate independently and it would enable them to take up GPS for independent navigation in unknown environment. The information derived from the research could further help in creating and improving the semantics of the GPS based navigation technology for the blind pedestrians in an unknown environment.
\end{abstract}

Keywords— independent navigation; semantics; GPS; pedestrians; visually impaired

\section{INTRODUCTION}

Being blind categorically is one of the extreme disabilities an individual may persist in a lifetime. In spite of numerous innovations to helping out this group of people to live normally, it still remains to be the most needful type of disability today. The scope of this research in particular is upon understanding and helping them how to navigate outdoors independently. People with vision disabilities are the most dependent group because they can't safeguard for themselves from perils nor can respond to external spurs without assisted identification about their surroundings. Safe navigation is the most challenging aspect for the blind to maintain. Though assistive technology is growing and has improved their mobility to a certain level, they still are at a substantial downside. It is because mobility without vision requires replacement for the loss visual input information to elude obstacles and hazards while navigating. They have comparatively less information about landmarks, headings and self-velocity information that is crucial for navigating even when they could be provided by external maps or verbal directions. These verbal directions are specific set of phrases which are useful to normal travelers on GPS Waze ${ }^{\circledR}$ e.g. "U turn". The benefit of the same directives however to a blind pedestrian remains debatable. For example, the semantics word "u turn" is sufficient to provide all important information for normal user to correct his path from what he sees; and such privileges are totally deprived from blind pedestrian. Even with the mobility assistances, the semantics are geometrically eccentric [1]. They lack of close guidance therefore, study on natural phrases or directions with them may lead to understanding a better way to comprehend their ways of directives hence to improve their navigation safety.

The endeavours in finding answers to effective mobility and and affordable too for the blind have been among the top priorities in the last couple of decades. The long-haul objective has been about to develop an independent and mobile framework which enables visually blind people to navigate independently and safely through acquainted and unacquainted environments. Smartphone concept has fulfilled half of the objective. They are basically electronic communication devices that can associate visually blind individuals with other innovative solutions and also easily available to them [2]. The current manoeuvres used by blind navigators are mainly tangibly dependent e.g a walking stick. Some came with sound directions notifying about surrounding obstacles, others are gadgets which can be stick fitted with hand-held or wearable gadgets to notify or warn the individual about obstacle ahead or serve as 'turn by turn' direction tool [3]. Some involve the GPS, vision 
substation system, sound signs, vision enhancement and optical recognition, obstacle detection and avoidance. Yet, the information between a blind pedestrian and the gadget remain tangible otherwise auditory or both.

\section{BACKGROUND}

World Health Organization (WHO) reported about 285 million people are visually impaired as of 2012 and out of that 246 million have low vision and 39 million are blind [4].This forms a large number of blind population around the world and formulates subjects to study for solutions through technological aspect. The mobility and navigation of a blind depend on their recognition and information of the environment that surrounds them. Global Positioning System (GPS) has been utilized by the visually impaired people for the purpose of navigation; as their movement and travelling solely depends on information and recognition of surroundings, the GPS based systems helps them to reach the destination and obtain relevant information about particular destination [5]. The blind pedestrians frequently use GPS based services and Global Positioning System in order to obtain appropriate and timely data applicable to a specific destination [16]. Despite the fact that various studies have shown the value and precision of GPS based route system for people with visual impairments information on the viability, potential and constraints of such devices in the users' common habitats is as yet inadequate [6]. For some, navigational assignments, a GPS-based individual guidance system for visually impeded individual's needs to give straightforward textual guidelines, for example, "go straight three pieces, turn left, and continue two blocks". Be that as it may, it is a plausibility to accept that a visually impaired tourist may likewise need something beyond textual data as a rule. For instance, a tourist would need to find a particular access to a shop or a building, a particular transport station or travel stop or a post box or pay telephone. In this way, the situating ought to be enhanced by telling the client the encompassing condition. For instance, the user can confirm the position of the walkway by telling him the location of the street and the buildings [7]. As for the route, data got through sound is imperative for building up a feeling of spatial orientation and distance along with obstacle recognition and evasion [8]. Most of the time blind people need exact and definite depiction of the environment, therefore, Semantic Schemata acts as a central processing unit to observe a psychological mapping of the surrounding for the individual. The declining expense of GPS components, combined with the current development in the accessibility of voice acknowledgement administrations, shows a chance to make a minimal effort arrangement. A important need of this framework is to meet the client's navigational needs while guaranteeing compactness and minimal effort.

A few sorts of regular helping strategies have been utilized to take care of this issue and the most broadly utilized technique is a white stick, which a person should learn completely to utilize. The other arrangement is a guide dog, which is exceptionally expensive however can't give so much information [9]. Numerous innovative solutions generally are known as Electronic Travel Aids (ETA) have onward been purposed and executed, however, none have been broadly effective in enhancing the movement and circumstances of the visually impaired [10]. According to previous studies, the use of GIS in blind navigation is that GIS, with their overwhelmingly visual correspondence of spatial data, may seem to have little to offer individuals with visual disabilities or visual impairment [11]. The diminishing expense of GPS units, combined with the current development in the accessibility of voice recognition system, introduces a chance to make a minimal effort solution. A key need of this framework is to meet the users' navigational requirements while guaranteeing minimal effort and convenience. Moreover, with a lot of advances in the GPS technology, the GPS receivers are built inside the mobile phones. GPS way finding frameworks are fundamentally appropriate for open-air conditions in light of the fact that the beneficiaries are regularly unfit to perform well in an indoor situation. Strategies for relative situating inside incorporate sensors utilizing digital tags, sonar, and accelerometers. A portion of the present GPS system influence utilization of Braille consoles for user input or output. Nonetheless, not all visually impeded individuals can read Braille [12]. Location-specific information is always helpful for the users to get aware of their location and landmarked information such as room numbers on a door, floor-plan, signage etc are parameterized to drive its significant information. [13]. In this unique situation, other than individual spatial data (e.g., area, course), the prerequisites of the visually impaired to a great extent incorporate basic geospatial data (e.g., the heading of the way, the passage of the building, and the areas of the intersection and transport stop), and points of interest of the particular data (e.g., the condition of the lamppost, the distance of the handrail, the width of the path, and the little scarp on the way). This descriptive information can help a visually impaired to form a mental map of the surrounding and facilitate the process of navigation to the desired destination.

The main drawback with the approaches are that they used by blind people for navigation is that solving the constraints and insufficiencies of the human misjudging because of the visual impairment is as yet an open issue. The difficulties engaged with planning a route system for visually impaired users are the way to consider a situation that upholds the imperatives of a genuine requirement for the safe route. Additionally to improve the impression of the client at each progression in light of a continuous expansion is a testing angle and to accentuate the semantic prompts induced from an important mapping can be a significant difficulty for the current navigational frameworks being used and proves to be a major setback for the current 
navigational systems in use by the visually impaired. In the present, civilian GPS fixes under a clear view of the sky are on average accurate to about 5 meters ( $16 \mathrm{ft}$ ) horizontally, which is a very big lag on the success of GPS amongst the blind users (derived from the experimental constraints).

Therefore, directing the travel route in terms of blind understandable units to notify the intended travel route and to predict a human walk and plan a path by avoiding the obstacles on the way towards the destination is the need of the hour. Therefore, the paper presents a way in which the blind can be assessed based on the usage of GPS and the information given to the blind by the narratives used in the GPS application. The effectiveness of the GPS while travelling outdoors would be assessed based on the parameters of time, distance errors and path deviation errors made while navigating using the GPS and while being guided by a human guide in an unknown environment outdoor. 1

\section{METHODOLOGY}

The experiments were done in two steps; the first step was conducted with an individual participant to check the feasibility of the experiments before recruiting the other set of multiple participants for the second study. For the multiple participant study, 10 participants were recruited with the criteria of being over the age of 21 , are experiencing blindness, use at least one navigation aid (white cane or a guide dog) because traditionally white cane is the most common and simplest tool used by the blind while navigating to scan obstacles on the ground such as holes, steps, walls, uneven surfaces etc. Some researchers have tried GPS technology in the past or are using a GPS based navigational applications, they should have experience in using technology and have basic orientation and mobility training [14]. In order to understand and observe the actions and describe the findings of this study. The research methodology used was an ethnographic study design. Ethnographic research is a qualitative method where researchers observe and/or interact with a study's participants in their real-life environment. The aim of an ethnographic study within a usability project is to get to know the reality of a design problem which was identified in the survey to find out the usage of GPS technology amongst the blind community in Malaysia (MFB). Thus, the ethnographic research design was suitable for this research where researcher is fully involved with participants and facilitates the researchers for documentation of the findings. This research needs to be conducted in a natural setting. It was done within the field sit and provided a holistic systematic overview of the context. The research was descriptive and interpretive and helped in documenting native perspectives. The research was carried out first as a pilot study to test the hypothesis and then as a multiple participant study with each participant in order to gather a clear understanding of their experience while navigating using GPS technology, as represented by the Fig 1. The steps involved were as follows: An observation trip on an unfamiliar route using a white cane, an observation trip on an unfamiliar route using GPS technology and a follow-up interview.

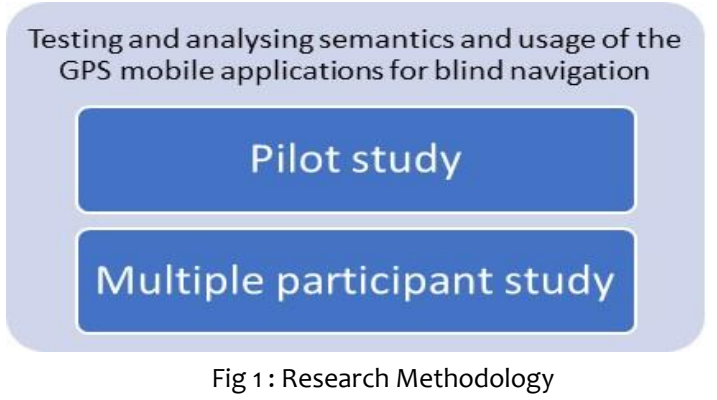

The GPS navigation technology was first tested within a single-participant pilot study. The main purpose of this study was to determine if the test would be feasible. The participant was required to perform three sets of navigation. In each set there were two runs, one run was performed with the help of guide directions and the other run was performed with the help of GPS instructions, however, the participant was carrying a white cane in both cases for his own comfort. The total number of runs were six, done in a set of three. In each set, the first run was taken using the verbal directions of the guide and the second run while returning from the same location was taken using the GPS navigation.

After completing the single-participant pilot study, a similar study involving 10 participants with a visual impairment was conducted. The participants were recruited by the blind foundation of Malaysia. The data was clarified and confirmed with participants through follow up interview. After that, the data was used to analyse and identify the benefits and limitations of navigation using a GPS application. The major stress was laid on the information lacking for the blind while navigating.

\section{RESULT AND DISCSSION}

The first hypothesis of the research suggests reasonably since the blind participants with the training of mobility orientation could navigate well with the help of verbal directions given to them by the human guide as shown in table 1.

TABLE I AVERAGE NUMBER OF DEVIATIONS/DISTANCE/TIME INSTRUCTIONS BY GUIDE

\begin{tabular}{|c|c|c|}
\hline $\begin{array}{c}\text { Average no. } \\
\text { of } \\
\text { deviations } \\
\text { (total no. of } \\
\text { deviations } / 3 \text { ) }\end{array}$ & $\begin{array}{c}\text { Average distance } \\
\text { error } \\
\text { (total no. of distance } \\
\text { increment } / 3 \text { ) }\end{array}$ & $\begin{array}{c}\text { Average time } \\
\text { taken } \\
\text { (Total time taken }\end{array}$ \\
\hline $5+7+6 / 3=6$ & $37+40+80 / 3=52$ meters & 13 ) \\
\hline
\end{tabular}


The same concept of giving instructions in natural language could be applied in the GPS applications while giving instructions to the blind people while navigating. The second hypotheses was backed up by the feedback of the blind participants that the verbal directions given by the GPS applications while navigating in an unknown environment are insufficient for a blind person to navigate with the confidence of safety and security. This point has also been laid emphasis on by previous researchers in which the blind demand more explanation to create a visual understanding of the routes and environment [15]. Therefore, the addition of an elaborate description of the route can help the blind users to take up GPS applications while navigating. The last hypothesis was based on a few parameters like the difference of time taken by the participants to navigate using GPS compared to guides instructions. The deviation errors made while navigating and the distance error made while navigating with and without GPS.

The common point mentioned by all participants was that the GPS technology is used only for outdoors as the signal indoors doesn't allow GPS to work well indoors. Therefore, validating the points made by [9] that outdoors, commercially the GPS can provide the information of the position within 20 meters accuracy. GPS is used on unfamiliar routes to give the blind an idea of their location and help them to create a mental map of the place as stated in a previous research [16]. However, while navigating there was an average error of 15 to 25-meter range as shown in Fig 2. Moreover, the GPS was getting affected by tall buildings. The blind people felt more information should have been provided to them by the GPS while navigating around. Timely instructions were not given which made the participants lack confidence using technology and felt unsafe as often found out in previous studies that the users need time to trust the system to use it independently [12]. The GPS application was unable to detect obstacles and barriers which is one the major concerns of the blind while navigating on their own. The same concern was mentioned in the research done earlier which mentioned that GPS fails to give obstacle detection and warning alerts [17].

In the event that courses are very much depicted for a given situation and accumulations, of course, portrayals are made accessible at that point the visually impaired will have an intense apparatus. University students who are new to some grounds could freely discover their classrooms. Travellers to urban areas could explore suitable destinations in a way like sighted people. The availability obstructions for visually impaired explorers would be definitely brought down.

Overall the finding of this study was validated by the previous research done on the visually impaired and their online route information management [14]. The main hypothesis was validated by the findings and the study shows that the blind are ready to take up GPS applications for navigation. Such works have been proved earlier [18] in which all the participants realized the advantages of the use of GPS based applications for outdoor navigation, however, stating that there were a lot of limitations.

The time taken by each participant while using the GPS was compared to the time taken while they were using their own traditional method of navigating. The distance covered each time was 1100 meters in each run. As shown on the map in Fig 6 and the average difference in time and distance in the multiple participant study is as shown in Fig 5 .

In their own traditional method, all of them used the white cane and asked the guide with them for the directions (one of the researchers) to ensure they were on the right track towards their destination. As shown in many earlier types of research [18]. That visually impaired people prefer to obtain navigational information and directions which are accessible and relevant to them, the sighted people can give good directions to the blind. However, while using the GPS they were asked to just follow the directions given to them by the GPS and not to any guide around them unless in danger. The difference in the time is shown in Fig 3. Moreover, there were deviations made by each participant while using the GPS based application which was counted as errors as shown in Fig 4. The participants stated that GPS eliminates navigating strategies used by blind people like counting steps or asking for directions unless the GPS stops working. The GPS provided with information about the location points in relation to current position.

The participants mentioned the use of natural language and more information would be really helpful for them in understanding the surroundings. As mentioned in previous research, people with visual impairments are capable of following natural language instructions similar to those given by a person communicating route directions [19]. In fact, in recent years, a large group of researchers have shown interest in sharing information about the surroundings for the blind people to navigate safely in an unknown environment [2]. Because of the lacking information on existing maps such as Google maps, open street map and other sources [20].

The GPS applications are beneficial for the blind users in terms of providing direction and independent mobility, however; the usage of GPS based applications still remains slow because of the limited features available in the existing applications. Even though the growth of usage of smartphones has rapidly increased in the blind communities as they have become more usable for the blind in terms of integration of functions needed as text to speech, gesture recognition and localization [21]. From Malaysian perspective, the applications can't fully help them in safely navigating as the roads are not constructed in a way to be navigated by the blind. So, the blind are not able to detect temporary obstacles similar to the idea proposed in a research previously [22] defining the concept of sharing points of interest, such as temporary obstacles for mobility 
of impaired pedestrians. So the information could be received by people based on user experience. By such sharing of information, the visually impaired could access, understand and explore the unfamiliar environment and improve their inclusion and improvement in the society [23]. The GPS verbal directions are limited and thereby the blind user feels a void while using the technology. Therefore, there should be an improvement in the GPS signals and explanation of the environment rather than just the descriptive information on how to navigate in real situations [12].

Further quantitative data helps to understand the acceptance and usage of the GPS technology amongst the blind. The use of GPS is a holistic approach and considers the environment it's used in as well as the skills of the user in orientation and mobility for effectively navigating., including a verbal route guidelines segment to a current GPS-based ETA would empower the gadget to give verbal route directions for indoor situations, areas that are not bolstered because of GPS's technical constraints. Indeed, even territories secured by the current gadgets could be supplemented by verbal course directions. For instance, numerous GPS gadgets essentially cover road-based routes because of the information wellspring of the maps they utilize. Verbal path portrayals could supplement open-air locations, for example, school grounds, doctor's facilities, workplaces, shopping malls' where there are substantial zones of walkways and couple of lanes

Moreover, many features such as semantics services customized to users' needs and preferences such a pathfinding, navigation and visualizing should be taken into account for the visually impaired [1], so the exploitation the information to guide the user should be according to users position and in that way the best route responsible for tracking users movement and giving useful information.

\section{CONCLUSION}

This paper explored the use of GPS mobile applications for the blind pedestrian navigation. The future work lies on the improvement of user trust on the GPS based applications in terms of accuracy and perceiving environmental information. The research helped to analyse and understand the use of technology for navigation by the blind people. The research also helped in laying emphasis on the lack of sufficient information given to the blind while navigating in an unfamiliar environment. It was also established that the use of correct phrases and units plays a very crucial role as all the participants relied heavily on the distance measuring units of the applications while being guided by the GPS based applications. All the blind participants demanded specific locations without any error to feel confident and safe while navigating. That can improve the effectiveness of the existing applications. The errors are in the distances while navigating was caused by the lack of understanding of the synthesized voice of the GPS. The speed of the blind people also slowed down while navigating using the GPS because of insufficient information given to them by the applications.

Therefore, if the existing GPS applications would improve the semantics of their narratives it would be profitable and the use of GPS based applications would prove to be advantageous to the community. So, the future work would include implementing control measures for avoiding repetition of instructions, improving accuracy of the GPS and giving precise explanation of the surroundings to the users.

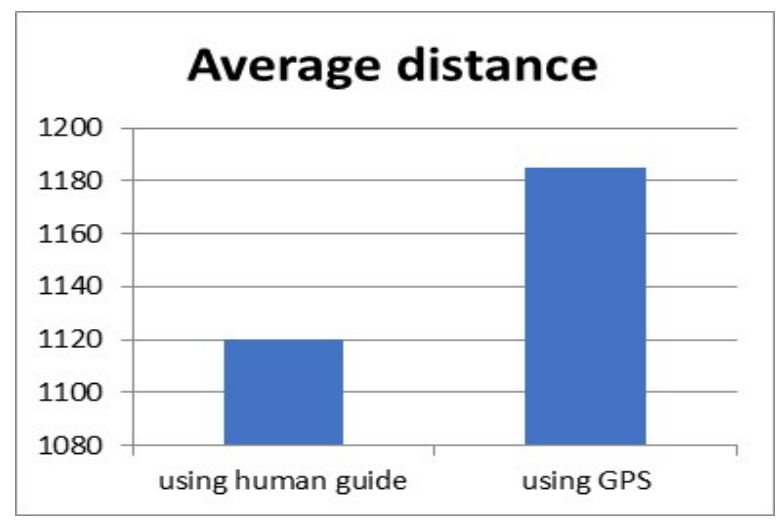

Fig 2: Error in meters shown for 10 participants

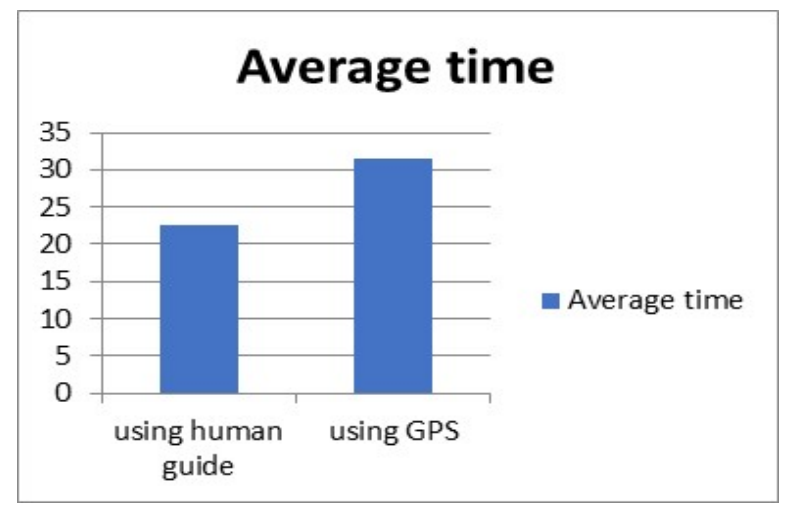

Fig 3: Time taken in minutes to navigate in minutes for 10 participants

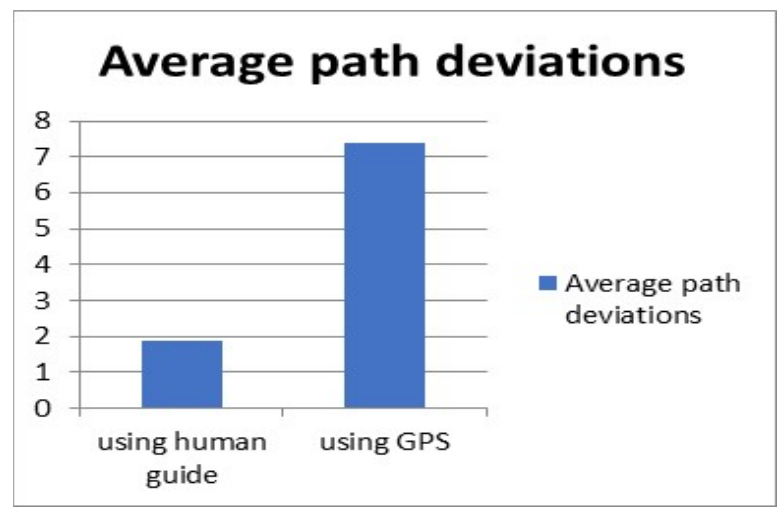

Fig 4: The number of deviations for 10 participants 


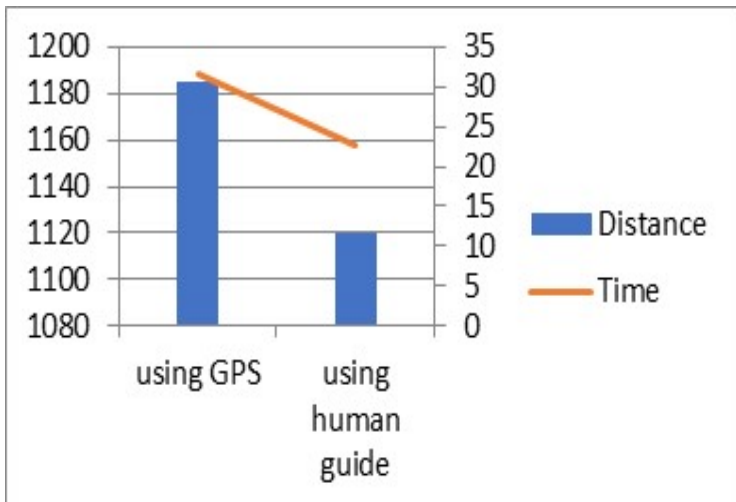

Fig 5: Difference between distance in meters and time minutes between using GPS and human guide(e) Map showing the distance

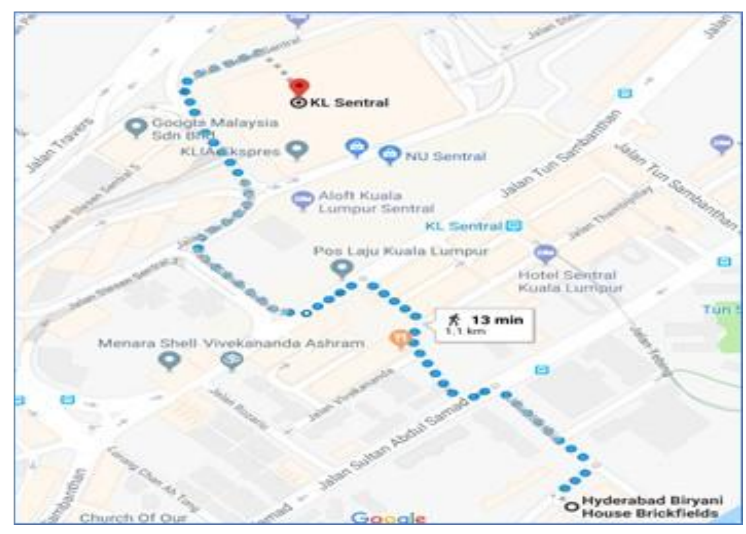

Fig 6: Map showing the distance

\section{Acknowledgment}

The authors would like to thank the MFB of Kuala Lumpur and the team who supported and participated all the way in this project. It also goes to the Research Initiative Grants Scheme (RIGS17-140-0715) of the IIUM, Gombak

\section{References}

[1] E.Nikoloudakis, M. Kritsotakis, A. Bikakis, T. Patkos, G. Antoniou and D. Plexousakis, "C-NGINE: A Contextual Navigation Guide for Indoor Environments," European Conference on Ambient Intelligence, Aml 2008: Ambient Intelligence, pp 258-275, 2009.

[2] B. Min, S. Saxena, A. Steinfeld, and M. Bernardine Dias, "Incorporating Information from Trusted Sources to Enhance Urban Navigation for Blind Travelers," Robotics and Automation (ICRA), 2015 IEEE International Conference, 2015.

[3] I. Shim and J. Yoon, "A robotic cane based on interactive technology," in IECON' 02 Industrial Electronics Society, IEEE 2002 28th AnnualConference, vol. 3, pp. 2249-2254, 2002.
[4] Visual impairment and blindness Fact Sheet N282, retrieved on Febuary 2015. http://www.who.int/mediacentre/factsheets/fs282/en.

[5] J.M. Loomis, J.R. Marston, R.G. Golledge and R.L. Klatzky, "Personal guidance system for people with visual impairment: A comparison of spatial displays for route guidance. Journal of Visual Impairment \& Blindness, 99 (4), 2005.

[6] P.E. Ponchillia, E.C. Rak, A.L. Freeland and S.J. LaGrow, "Accessible GPS: Reorientation and target location among users with visual impairments," Journal of Visual Impairment \& Blindness, 101 (7), 2007.

[7] Markus et al., "Comparison of Open Source routing services with OpenStreetMap Data for blind pedestrians," Markus Dornhofer, Werner Bischof, ElmarKrajnc, 2014.

[8] J.J. Rieser, "Blindness and brain plasticity in Navigation and object perception," New York, NY: Lawrence Erlbaum Associates/Taylor Francis Group, 2008.

[9] M. B. Salah and M. Fezari, "The Development of a Pedestrian Navigation Aid for the Blind" 2010

[10] G. Ghiani, B. Leporini and B. Paterno, "Vibrotactile feedback to aid blind users of mobile guides," J. Vis. Lang. Comput. 20, 305-317, 2009.

[11] Kitchin and Jacobson, "GIS and people with visual impairments or blindness: Exploring the potential for education, orientation, and navigation," R DAN JACOBSON AND ROBERT M KITCHIN, 1997.

[12] Somnath and Mishra, "Voice Operated Outdoor Navigation System for Visually Impaired Persons," 2012.

[13] Samleo, Joseph, Z. Xiaochen, D. Ivan and X. Jizhong, "Semantic indoor navigation with a blind-useroriented augmented reality," 2013.

[14] [Review Paper on Navigation System for Visually Impaired People Chaitali K. Lakde1, Dr. Prakash S. Prasad 2015]

[15] K. Vladimir, J.Nicolson and J.Martson, "The Blind Leading the Blind: Toward Collaborative Online Route Information Management by Individuals with Visual Impairments,"'Laboratoired'Eco-Anthropologie et d'Ethnobiologie (CNRS-MNHN-ParisVII) Paris, France, 2010.

[16] N. Justason, J. Treviranus, L. McArthur and J. Silva, "GPS-based navigation in the everyday life of blind users," Department of Occupational Science \& Occupational Therapy, University of Toronto, 2010 .

[17] C. K. Lakde and P. S. Prasad, "Review Paper on Navigation System for Visually Impaired People," IJSRSET, Volume 2 .2, pp. 2394-4099, 2016

[18] J. Balata, J. Franc, Z. Mikovec, and P. Slavik, "Collaborative navigation of visually impaired," Journal of Multimodal User Interfaces, vol. 8, no. 2, pp. 175-185, 2014.

[19] J. Nicholson, "Generation and Analysis of Verbal Route Directions for Blind Navigation," Utah State University, 2010.

[20] J. M. Coughlan and H. Shen, "Crosswatch: a System for Providing Guidance to Visually Impaired Travelers at Traffic Intersections," JAssist Technol, vol. 7, no. 2, 2013.

[21] A. Smith, "Nearly half of American adults are smartphone owners," Pew Research Center's Internet \& American Life Project, 2012.

[22] T. V“olkel, R. K"uhn, and G. Weber, "Mobility impaired pedestrians are not cars: Requirements for the annotation of geographical data", ICCHP '08 Proceedings of the 11th international conference on Computers Helping People with Special Needs, pp. 1085-1092, 2008.

[23] X. Zhang, B. Li, S. L. Joseph, J. Xiao, Y. Sun and Y. Tian, "A SLAM based Semantic Indoor Navigation System for Visually Impaired Users," The City University of New York New York, USA, 2015. 